\title{
Physicochimie et biochimie des protéines du lait. Données récentes
}

\author{
B Ribadeau-Dumas
}

I NRA, station de recherches laitières 78352 Jouy-en-Josas cedex, France

\begin{abstract}
Résumé - Après un bref rappel sur les protéines en général, cette revue s'attache à décrire les découvertes les plus récentes (10 dernières années) concernant les protéines du lait, principalement d'origines humaine et bovine. Elle voudrait conduire les industriels laitiers à tenter d'utiliser ces protéines en fonction de leurs activités biologiques.
\end{abstract}

protéine du lait / structure / activité biologique

Summary - Physicochemistry and biochemistry of milk proteins. Recent results. After a brief overview of proteins in general, this review describes the most recent discoveries (over the last $10 \mathrm{yr}$ ) mainly concerning human and bovine milk proteins. The aim is to encourage dairy firms to try to use these proteins according to their biological functions.

milk protein / protein structure / biological activity

\section{INTRODUCTION : LES PROTÉINES}

Une revue sur les protéines du lait dans le cadre de la santé appelle un bref rappel sur les protéines en général.

Celles-ci comprennent une ou plusieurs chaînes linéaires dont les éléments constitutifs sont 20 acides aminés différents (résidus, les chaînes comportant 60-5000 résidus. Dix des 20 acides aminés constitutifs sont essentiels : ils ne peuvent pas être synthétisés par l'organisme à partir d'autres molécules. Un dosage d'acides aminés simple ne fournit que 16 valeurs: $\mathrm{Asp}^{*}+$ Asn $\rightarrow$ Asp, Glu + Gln $\longrightarrow$ Glu; Cys et Trp sont détruits. La distinction entre acides aminés acides (Asp et Glu) et leurs amides (Asn et Gln) ne porte pas à conséquence au plan nutritionnel : aucun des 4 n'est essentiel. Par contre la cystéine et le tryptophane le sont. Ils nécessitent chacun un dosage particulier.

Au plan de la structure des protéines, les caractéristiques des chaînes latérales qui différencient les 20 acides aminés sont essentielles. Certaines ont une charge électrique, positive ou négative, qui dépend du $\mathrm{pH}$. D'autres possèdent une structure dipolaire mais n'ont pas de charge nette (elles renferment un ou plusieurs atomes d'azote et/ou d'oxygène). D'autres enfin, ne sont constituées que de carbone et d'hydrogène. Étant apolaires, elles n'ont aucune affinité pour les molé-

\footnotetext{
${ }^{*}$ Asp = acide aspartique; Asn = asparagine; Glu = acide glutamique; Gln = glutamine; Cys = cystéine; $\operatorname{Trp}=$ tryptophane; $\operatorname{Tyr}=$ tyrosine; Met = méthionine.
} 
cules polaires de l'eau. Bien que leur chaînes latérales contiennent oxygène, azote ou soufre, 3 acides aminés (Tyr, Trp, Met) sont également apolaires. Le repliement de la chaîne peptidique qui donne la protéine native est dicté par l'enchaînement des acides aminés. Les acides aminés apolaires se regroupent à l'intérieur, à l'abri du solvant. Les acides aminés polaires se trouvent à l'extérieur, en contact avec le solvant. La rigidité de l'édifice, le plus souvent très compact, est parfois accrue par la formation de ponts disulfures entre résidus de cystéine. Sur la surface de la protéine sont souvent fixées des chaînes glucidiques, parfois des groupements phosphate. Les sites antigéniques ou allergéniques d'une protéine sont des motifs, constitués de 7-8 résidus d'acides aminés proches, situés à la surface de la protéine et reconnus par des anticorps. Deux techniques seulement permettent de caractériser dans ses moindres détails la structure tridimensionnelle des protéines : la diffraction des rayons $X$ et la résonance magnétique nucléaire. La première exige l'obtention de "bons" cristaux; la deuxième, qui étudie les protéines en solution, n'est actuellement applicable qu'à des molécules de masse moléculaire inférieure à $15000 \mathrm{Da}$.

On admet que chaque protéine a une fonction biologique. Deux protéines appartenant chacune à une espèce différente, localisées dans des cellules homologues ayant la même fonction, sont en général homologues: les séquences de leurs acides aminés sont plus ou moins proches ; leurs structures tridimensionnelles sont très voisines. Plus les espèces sont éloignées, plus les séquences de protéines homologues sont différentes, mais les structures tridimensionnelles restent voisines. Chez les individus d'une même espèce, les protéines sont identiques, ou ne diffèrent dans leur séquence que, généralement, par la substitution de 1 ou de 2 ré- sidus par 1 ou 2 résidus différents. On a affaire à des variants génétiques.

\section{LES PROTÉINES MAJEURES DU LAIT}

Que connaît-on de la structure des protéines du lait de vache et de femme? Sont connues les structures primaires des 6 protéines bovines majeures (les 4 caséines, l' $\alpha$-lactalbumine $-\alpha$ La, la $\beta$ lactoglobuline - $\beta \mathrm{Lg}$ ) (Swaisgood, 1982; et corrections dans Ribadeau-Dumas, 1988), de la butyrophiline (Jack et Matter, 1990) et des lactoperoxidases bovine et humaine (Dull et al, 1990; Cals et al, 1990); des 2 caséines humaines ( $\beta$ et $\kappa$ ) (Brignon et al, 1985; Lönnerdal et al, 1990), de l' $\alpha$ lactalbumine (Hall et al, 1982), de la lactoferrine (Powell et Ogden, 1990; Rey et al, 1990) et du lysozyme (Canfield et al, 1971; Jollès et Jollès, 1971) humains. Sont connues également les structures tridimensionnelles de l' $\alpha$-lactalbumine de babouin, très voisine de la protéine humaine (Acharya et al, 1989), de la $\beta$-lactoglobuline bovine (Papiz et al, 1986; Monaco et al, 1987), du lysozyme et de la lactoferrine (Anderson et al, 1989) humains.

À cause, probablement, de leur teneur élevée en acides aminés hydrophobes et en proline, les caséines s'agrègent en solution et forment des structures beaucoup plus lâches que la plupart des autres protéines. Aucune n'a pu être cristallisée. On peut d'ailleurs penser qu'il faudra longtemps avant qu'il soit possible de déterminer la structure des caséines dans leur état natif, c'est-à-dire en interaction entre elles et avec le phosphate de calcium, comme elles le sont dans les micelles.

Quelles sont les avancées majeures des 10 dernières années concernant la physicochimie des protéines du lait ? Elles sont certainement moins importantes que celles des années 70 . 


\section{Caséines}

En ce qui les concerne, la biologie moléculaire a permis de déterminer indirectement leur structure primaire chez de nombreuses espèces, notamment des animaux de laboratoire : souris, rat, lapin, (Ribadeau-Dumas, 1988). Une chose apparaît très clairement : les caséines ont évolué très rapidement. Seuls sont conservés les segments polyphosphorylés des caséines $\alpha_{s 1}, \alpha_{s 2}, \beta$ et le segment qui suit immédiatement, dans la caséine $\kappa$, la liaison coupée par la présure. L'hydrophobicité élevée et la richesse en proline sont également conservées. II apparaît par ailleurs que les proportions des caséines $\alpha_{s 1}, \alpha_{s 2}$ et $\beta$ varient considérablement d'une espèce à l'autre, et même à l'intérieur d'une même espèce (caprins : Grosclaude et al, 1987).

On peut en conclure que ces 3 caséines sont interchangeables, qu'elles ont des caractéristiques d'agrégation et de fixation du phosphate ( $\mathrm{Ca}$ et $\mathrm{Mg}$ ) uniques. Ceci permet, dans un volume minimal (certains laits contiennent plus de $100 \mathrm{~g} / \mathrm{l}$ de protéines), d'apporter simultanément au jeune une quantité élevée de protéines facilement digérées, de phosphate et de calcium.

II est clair également que les caractéristiques du caillé, dans la cuve du fromager ou dans l'estomac, à teneurs en caséines égales, dépend essentiellement du degré de phosphorylation des caséines. Une déphosphorylation partielle des caséines dans le lait de vache permet d'approcher la structure du caillé de lait humain dans l'estomac du nourrisson (Li-Chan et Nakai, 1989).

\section{Protéines du lactosérum}

Dans ce domaine des avancées majeures ont eu lieu grâce à l'élucidation de la struc- ture tridimensionnelle, de la $\beta$-lactoglobuline $(\beta \mathrm{Lg})$, I' $\alpha$-lactalbumine ( $\alpha \mathrm{La}$ ) et la lactoferrine (LF) et à la détermination de la structure primaire de la lactoperoxydase (LP).

\section{$\beta$-lactoglobuline}

La $\beta$-lactoglobuline a une structure très voisine de celle de 6 ou 7 protéines ayant toutes pour fonction la fixation et le transport d'une petite molécule hydrophobe. On savait depuis longtemps que la $\beta \mathrm{Lg}$ fixait énergiquement le rétinol, précurseur de la vitamine A. La présence, dans l'intestin du jeune veau, de récepteurs à la $\beta \mathrm{Lg}$ qui disparaissent au sevrage a été montrée (Papiz et al, 1986), de même qu'a été montré l'effet stimulant de la $\beta \mathrm{Lg}$ sur l'absorption intestinale du rétinol chez le rat non sevré et adulte (Said et al, 1989). Mais des points restent obscurs quant à une possible implication de la $\beta \mathrm{Lg}$ dans l'absorption du rétinol. Peut-être une protéine homologue endogène existe-t-elle, qui remplacerait la $\beta \mathrm{Lg}$ chez l'adulte et chez le jeune des espèces dépourvues de $\beta \mathrm{Lg}$. La $\beta \mathrm{Lg}$ a été mise en évidence dans tous les laits de ruminants étudiés, ainsi que chez 7 monogastriques : truie, jument, ânesse, chienne, dauphin, lamentin, kangourou. Chez les ruminants et le kangourou, elle se trouve à l'état dimérique dans le lait, chez les 6 autres espèces monogastriques, à l'état monomérique. Les laits de jument et d'anesse contiennent chacun 2 $\beta \mathrm{Lg}$ différentes, dont l'une, contrairement à toutes les autres $\beta \mathrm{Lg}$, n'a pas de groupement thiol libre (Godovac-Zimmermann et al, 1990).

\section{$\alpha$-lactalbumine}

Le rôle physiologique majeur de l' $\alpha$ lactalbumine dans la biosynthèse du lactose est bien connu. Sa structure tridimen- 
sionnelle a permis de mieux comprendre l'importance de l'ion calcium qu'elle renferme, et ce qui la distingue du lysozyme. On savait en effet depuis longtemps que ces 2 protéines avaient des structures voisines, tout en ayant des fonctions différentes. On sait maintenant que l'ion $\mathrm{Ca}^{2+}$ est essentiel au repliement de la chaîne peptidique, au maintien de sa structure native (Rao et Brew, 1989). Le passage de I' $\alpha$ La en-dessous de $\mathrm{pH} 3,5$, qui entraîne l'élimination de cet ion, produit une protéine semi-dénaturée, insoluble dans une assez large gamme de $\mathrm{pH}$ mais rapidement digérée par la pepsine qui n'attaque pas l' $\alpha$ La native au-dessus de ce $\mathrm{pH}$ (Miranda et al, 1989).

L'ion $\mathrm{Ca}^{2}+$ est entouré de plusieurs carboxylates (chaînes latérales de plusieurs Asp) chargés négativement. Son élimination entraîne une répulsion électrostatique de ces derniers, et une ouverture de la molécule. Ces carboxylates ne sont pas présents dans la plupart des lysosymes. Ils le sont dans les lysozymes de jument, d'ânesse et de pigeon (voir dans Acharya et al, 1989) qui fixent le calcium.

\section{Lactoferrine}

La détermination de la structure tridimensionnelle de la lactoferrine humaine a apporté des renseignements intéressants quant au mode de liaison du fer et de l'ion carbonate à la protéine. II semble confirmé que l'action bactériostatique de la LF n'est optimale qu'en présence d'une quantité suffisante de carbonate et d'une quantité minimale de citrate. Ces conditions sont beaucoup mieux remplies dans la mamelle, surtout en involution, que dans le tube digestif du nouveau-né. Par contre, la mise en évidence de récepteurs à la LF dans le tube digestif chez plusieurs espèces (Hu et al, 1988) pourrait donner à penser que la LF du lait, prise en relai en- suite par la LF intestinale, permet une récupération et une assimilation optimales du fer dans l'intestin. II existe une homologie de structure étroite entre la LF, la transferrine sérique, la conalbumine (ovotransferrine) et la mélanotransferrine de cellules de mélanome humain (Rose et al, 1986). Toutes ces protéines ont des propriétés similaires, notamment en ce qui concerne la fixation du fer et l'activité bactériostatique. Par contre, une similarité de structure curieuse a été observée entre ces protéines et celles de protéines bactériennes liant l'arabinose, la galactose, la leucine, l'isoleucine, la valine et le sulfate. La «sulfate binding protein» de Salmonella typhimurium présente l'homologie la plus étroite (Anderson et al, 1989).

\section{Lactoperoxydase}

La structure primaire de la lactoperoxydase bovine vient d'être élucidée, directement dans notre laboratoire, par l'intermédiaire de I'ADNc dans un laboratoire américain qui a également déterminé la séquence de la LP humaine, enzyme également présente dans le lait humain. La LP est étudiée depuis fort longtemps. Comme la $L F$, les lgAs et le lysozyme, on la trouve (ou tout au moins une peroxydase voisine) dans toutes les sécrétions externes. Elle joue un rôle bactéricide bien connu en catalysant l'oxydation par $\mathrm{H}_{2} \mathrm{O}_{2}$ de l'ion SCN, qui provient du catabolisme des acides aminés soufrés et de glucosides venant de l'alimentation.

Le second substrat $\mathrm{H}_{2} \mathrm{O}_{2}$, peut venir de diverses voies métaboliques, par exemple de l'action de la xanthine oxydase, enzyme de la membrane des globules gras. II est excrété par les bactéries catalasé comme les bactéries lactiques. L'oxydation de l'ion thiocyanate conduit à la formation de l'ion hypothiocyanate $\mathrm{OSCN}^{-}$, qui semble inactiver les bactéries Gram- sensibles par 
oxydation de thiols essentiels. La LP présente $45-55 \%$ d'identité avec 3 autres enzymes de mammifères : la myéloperoxydase, l'éosinophile peroxydase et la thyroïde peroxydase. Les 2 premiers sont relargués aux sites d'inflammation par les leucocytes neutrophiles et éosinophiles respectivement. La première oxyde l'ion $\mathrm{Cl}^{-}$en $\mathrm{OCl}^{-}$(hypochlorite; eau de Javel), la seconde, l'ion $\mathrm{Br}$ en $\mathrm{OBr}$ - - tous 2 agents bactéricides bien connus. La $3^{e}$ oxyde l'iodure en iodant les résidus tyrosyles de la thyroglobuline, précurseur des hormones thyroïdiennes. La LP est d'ailleurs également capable d'utiliser Icomme substrat : l'enzyme est couramment utilisée pour marquer les protéines à l'iode radioactif. Les 4 péroxydases en question ont comme cofacteur un hème dont le fer est trivalent, qui semble être fixé à l'enzyme par liaison covalente.

\section{LES PROTÉINES MINEURES DU LAIT}

Un certain nombre de protéines quantitativement mineures ont été isolées du lait et plus ou moins caractérisées. Nous ne parlerons ici que de celles qui le sont le mieux.

\section{Xanthine oxydase}

La xanthine oxydase (XO), qui est l'enzyme la plus abondante dans le lait de vache, est localisée en abondance à la face interne de la membrane des globules gras, dans les cellules secrétrices mammaires et dans les cellules endothéliales des capillaires sanguins. Elle se trouve, en faible quantité, dans toutes les autres cellules. C'est une grosse protéine, un dimère constitué de 2 sous-unités identiques de masse moléculaire $150 \mathrm{kDa}$. Chaque sous-unité contient comme cofacteur une molybdoptérine, comme la nitrate réductase et la sulfite oxydase, un centre flavine adénine dinucléotide (FAD) et 2 centres fer-soufre. La fonction principale de la XO dans toutes les cellules est liée au catabolisme des purines (adénine, guanine). L'enzyme catalyse en effet l'oxydation de l'hypoxanthine en xanthine et de la xanthine en acide urique. L'acide urique est excrété chez l'homme, et, chez les autres mammifères transformé en allantoïne qui est alors le produit final de dégradation des purines. L'hypoxanthine et la xanthine sont issues de l'oxydation directe respectivement de l'adénine et de la guanine. La fonction postulée de la $X O$ dans les tissus où elle est présente en abondance est liée à la modification de la structure de la membrane plasmique permettant l'exo- ou l'endocytose dans la glande mammaire (excrétion des globules gras) ou les capillaires. Elle pourrait également, chez le jeune, jouer un rôle dans l'absorption intestinale. La XO peut être responsable de défauts de goût dans les produits laitiers, surtout en présence d'ions cuivriques, en produisant une oxydation des lipides.

En effet les 2 réactions catalysées par la XO libérent à la fois de l'eau oxygénée et le radical superoxyde $\mathrm{O}_{2}^{-}$. L'eau oxygénée libérée peut d'ailleurs activer le système lactoperoxydase du lait (Bjorck et Claesson, 1979). La xanthine oxydase du lait, surtout lorsqu'il est homogénéisé, a longtemps été accusée de favoriser l'apparition de plaques d'athérome. Une fraction de l'enzyme passerait intacte dans le sang et catalyserait par endroit la dégradation des plasmalogènes des parois artérielles (Clifford et al, 1983).

Une lipase stimulée par les sels biliaires (BSSL) existe en abondance ( $1 \%$ des protéines totales) dans le lait humain. Cette enzyme est une lipase non spécifique qui hydrolyse les mono-, di- et triacylglycérols, les lysophosphatidyl glycérols, les esters 
du cholestérol et du rétinol. La détermination récente de sa structure primaire (Nilsson et al, 1990) a montré que la BSSL était identique à la carboxylic ester hydrolase du suc pancréatique humain, qui a la même fonction, et très homologue de l'acétylcholinestérase.

\section{CONCLUSION}

Si l'on considère à la fois les propriétés physico-chimiques des diverses protéines du lait et leurs fonctions biologiques, il commence à être possible d'envisager, au moins pour certaines d'entre elles, des applications spécifiques basées sur ce qui suit :

- les caséines, de par leur structure, ont des propriétés adhésives bien connues et sont incoagulables par la chaleur; elles peuvent fixer des quantités importantes de divers phosphates alcalinoterreux; elles sont facilement dégradées en courts peptides sous l'action de diverses protéases ; certains des peptides libérés ont des activités biologiques;

- la $\beta \mathrm{Lg}$ et l' $\alpha \mathrm{La}$, de par leur structure, ont des propriétés fonctionnelles intéressantes; celles-ci sont-elles suffisamment différentes pour que leur séparation ait un intérêt économique ? Ceci reste à montrer; d'un autre côté la première apparaît avoir une forte affinité pour de petits ligands hydrophobes, insolubles dans l'eau; ceci pourrait être mis à profit; de même, ne pourrait-on pas envisager d'utiliser l' $\alpha$ La pour la synthèse de molécules voisines, mais différentes du lactose?

- la fonction biologique de la LP dicte pratiquement ses applications; par contre, I'utilisation de la LF reste discutée ; cependant, sa très forte affinité pour le fer dans une large gamme de $\mathrm{pH}$ pourrait être exploitée;
- la xanthine oxydase peut activer le système LP.

Pour terminer, il est intéressant de noter qu'il a été trouvé très récemment que les différentes caséines sont synthétisées par les lymphocytes $T$ cytotoxiques chez la souris, et qu'on a postulé qu'elles joueraient un rôle indirect dans la cytotoxicité (Grusby et al, 1990).

\section{RÉFÉRENCES}

Acharya KR, Stuart DI, Walker NPC, Lewis M, Phillips DC (1989) Refined structure of baboon $\alpha$-lactalbumin at $1.7 \AA$ resolution. J Mol Biol 208, 99-127

Anderson BF, Baker HM, Norris GE, Rice DN, Baker EN (1989) Structure of human lactoferrin: crystallographic structure analysis and refinement at $2.8 \AA$ resolution. $J \mathrm{Mol} B i o l 209$, 711-734

Björck L, Claesson O (1979) Xanthine oxidase as a source of hydrogen peroxide for the lactoperoxidase system in milk. $J$ Dairy Sci 62 , 1211-1215

Brignon G, Chtourou A, Ribadeau-Dumas B (1985) Preparation and amino acid sequence of human k-casein. FEBS Lett 188, 48-54

Cals MM, Mailliart P, Brignon G, Anglade P, Ribadeau-Dumas B (1990) Primary structure of bovine lactoperoxidase, the fourth number of mammalian heme peroxidase family. Eur $J$ Biochem (sous presse)

Canfield RE, Kammerman S, Sobel JH, Morgan FJ (1971) Primary structure of lysozymes from man and goose. Nature New Biol 232, 16-17

Clifford AJ, Ho CY, Swenerton H (1983) Homogenized bovine milk xanthine oxidase: a critique of the hypothesis relating to plasmalogen depletion and cardiovascular disease. Am J Clin Nutr 38, 327-332

Dull TJ, Uyeda C, Strosberg AD, Nedwin G, Seilhaner JJ (1990) Molecular cloning of cDNAs encoding bovine and human lactoperoxidase. DNA Cell Biol 9, 499-509

Godovac-Zimmermann J, Conti A, Sheil M, Napolitano L (1990) Covalent structure of the 
minor monomeric $\beta$-lactoglobulin II component from donkey milk. Biol Chem HoppeSeyler 371, 871-879

Grosclaude F, Mahé MF, Brignon G, Di Stasio $L$, Jeunet $R$ (1987) A Mendelian polymorphism underlying quantitative variations of goat $\alpha_{s 1}$-caseins. Génét Sél Évol 19, 399412

Grusby MJ, Mitchell SC, Nabavi N, Glimcher LH (1990) Casein expression in cytotoxic T lymphocytes. Proc Natl Acad Sci USA 87, 6897. 6901

Hall L, Craig RK, Edbrooke MR, Campbell PN (1982) Comparison of the nucleotide sequence of cloned human and guinea-pig pre$\alpha$-lactalbumin cDNA with that of chick prelysozyme cDNA suggests evolution from a common ancestral gene. Nucleic Acids Res 10, 3503-3515

Hu WL, Mazurier J, Sawatzki G, Montreuil J, Spik G (1988) Lactotransferrin receptor of mouse small-intestinal brush border. Biochem J 249, 435-441

Jack LJW, Mather IH (1990) Cloning and analysis of cDNA encoding bovine butyrophilin, an apical glycoprotein expressed in mammary tissue and secreted in association with the milk-fat globule membrane during lactation. J Biol Chem 265, 14481-14486

Jollès J, Jollès P (1971) Human milk lysozyme: unpublished data concerning the establishment of the complete primary structure; comparison with lysozymes of various origins. Helv Chim Acta 54, 2668-2675

Li-Chan E, Nakai S (1989) Enzymatic dephosphorylation of bovine casein to improve acid clotting properties and digestibility for infant formula. J Dairy Res 56, 381-390

Lönnerdal B, Bergström S, Andersson Y, Hjalmarsson K, Sundqvist AK, Hernell O (1990) Cloning and sequencing of a cDNA encoding human milk $\beta$-casein. FEBS Lett 269, 153156

Miranda G, Hazé G, Scanff P, Pélissier JP (1989) Hydrolysis of $\alpha$-lactalbumin by chymosin and pepsin. Effect of conformation and pH. Lait 69, 451-459
Monaco $\mathrm{HL}$, Zanotti G, Spadon $\mathrm{P}$, Bolognesi M, Sawyer L, Eliopoulos EE (1987) Crystal structure of the trigonal form of bovine $\beta$ lactoglobulin and of its complex with retinol at $2.5 \AA ̊$ resolution. J Mol Biol 197, 695-706

Nilsson J, Bläckberg L, Carlsson P, Enerbäck S, Hernell O, Bjursell G (1990) cDNA cloning of human-milk bile-salt-stimulated lipase and evidence for its identity to pancreatic carboxylic ester hydrolase. Eur J Biochem 192, 543-550

Papiz MZ, Sawyer L, Eliopoulos EE, Worth $A C T$, Findlay JBC, Sivaprasadarao $R$, Jones TA, Newcomer ME, Kraulis PJ (1986) The structure of $\beta$-lactoglobulin and its similarity to plasma retinol-binding protein. Nature (Lond) 324, 383-385

Powell MJ, Ogden JE (1990) Nucleotide sequence of human lactoferrin cDNA. Nucleic Acids Res 18, 4013

Rao KR, Brew K (1989) Calcium regulates folding and disulfide-bond formation in $\alpha$ lactalbumin. Biochem Biophys Res Commun 163, 1390-1396

Rey MW, Woloshuk SL, de Boer HA, Pieper FR (1990) Complete nucleotide sequence of human mammary gland lactoferrin. Nucleic Acids Res 18, 5288

Ribadeau-Dumas B (1988) Structure and variability of milk proteins. In: Milk Proteins (CA Barth, E Schlimme, eds) Springer-Verlag, New York

Rose TM, Plowman GD, Teplow DB, Dreyer WJ, Hellström KE, Brown JP (1986) Primary structure of the human melanoma-associated antigen P97 (melanotransferrin) deduced from the mRNA sequence. Proc Natl Acad Sci USA 83, 1261-1265

Said HM, Ong DE, Shingleton L (1989) Intestinal uptake of retinol: enhancement by bovine milk $\beta$-lactoglobulin. Am J Clin Nutr 49,690694

Swaisgood HA (1982) Chemistry of milk proteins. In: Developments in Dairy Chemistry, vol 1 (PF Fox, ed) Appl Sci Publ, London 